\title{
Structural Changes of the Urinary Bladder After Chronic Complete Spinal Cord Injury in Minipigs
}

\author{
Elena E. Foditsch ${ }^{1,2}$, Karin Roider ${ }^{1,2}$, Irina Patras ${ }^{3}$, Ioan Hutu ${ }^{3}$, Sophina Bauer ${ }^{4}$, Günter Janetschek ${ }^{1}$, Reinhold Zimmermann ${ }^{1,4}$ \\ ${ }^{1}$ Spinal Cord Injury and Tissue Regeneration Center Salzburg, Paracelsus Medical University, Salzburg, Austria \\ ${ }^{2}$ Institute for Molecular Regenerative Medicine, Paracelsus Medical University, Salzburg, Austria \\ ${ }^{3}$ Banat University of Agricultural Sciences and Veterinary Medicine, Timisoara, Romania \\ ${ }^{4}$ University Clinics of Urology and Andrology, General Hospital Salzburg, Paracelsus Medical University, Salzburg, Austria
}

Purpose: The aim of this study was to determine the structural changes of the urinary bladder after chronic spinal cord injury (SCI) in minipigs with the primary focus on the analysis of urinary bladder wall proteins and their quantitative distribution. Methods: Seven Göttingen minipigs (adult, female) underwent a complete spinal cord transection. Follow-up time was 4 months during which the bladder was drained by frequent single catheterisation and data from the bladder diary and daily urine strip test were collected. Samples from the urinary bladder were taken, fixed in $4 \%$ paraformaldehyde and stained for histological analyses. Bladder wall thickness, single tissue quantities/distributions, types I and III collagen, and elastin quantifications were performed. Comparisons to healthy urinary bladder tissue of age-matched minipigs were performed for statistical analyses.

Results: No urinary tract infections were observed in our SCI minipig collective during follow-up. A trend towards a reduction in bladder volumes and an increase in incontinence periods were seen. The bladder wall thickness significantly increased after chronic SCI. Furthermore, bladder wall composition was severely altered by a significant loss of smooth muscle tissue and a significant increase in connective tissue. Elastic fibres were reduced in number and altered in their structural appearance after SCI. Type I collagen was significantly increased, while type III collagen was significantly decreased after SCI.

Conclusions: Chronic SCI highlighted that the urinary bladder wall undergoes fibrotic events with reduced contractile and elastic properties due to changes of the bladder wall protein composition. These changes show in detail how SCI severely influences the urinary bladder wall composition and depicts the similarities between minipigs and humans.

Keywords: Spinal Cord; Transection; Collagen; Elastin; Models, Animal

- Research Ethics: This study (Research Project No. 1, 10.10.2012) was carried out under the protocols approved by the Ethics and Deontology Committee for Research on Animals Victor Babes University of Medicine, Timisoara, Romania, and were in compliance with the Association for Assessment of Laboratory Animal Care guidelines for animal use.

- Conflict of Interest: No potential conflict of interest relevant to this article was reported.

\section{- HIGHLIGHTS}

- Chronic spinal cord injury changes the composition of urinary bladder wall proteins leading to a reduction in its contractile and elastic properties.

- The observed fibrotic changes show in detail how severe spinal cord injury influences the structure of the urinary bladder wall in minipigs.

Corresponding author: Elena Esra Foditsch (iD http://orcid.org/0000-0003-4560-1515 Department of Urology and Andrology, Spinal Cord Injury and Tissue Regeneration Center Salzburg, Paracelsus Medical University, Strubergasse 21, 5020 Salzburg, Austria

E-mail: esra.foditsch@pmu.ac.at / Tel: +43 662242080895 / Fax: +43(0)5 725527497 Submitted: July 15, 2016 / Accepted after revision: November 28, 2016 


\section{INTRODUCTION}

The storage and periodic elimination of urine are the 2 primary functions of the lower urinary tract (LUT) and involve a very complex interaction between the anatomic parts of the LUT and the nervous control systems [1]. The considerable complexity of regulatory neuronal mechanisms enables that the LUT function is sensitive to a variety of dysfunctions. Such is the case of spinal cord injury (SCI), which is associated with increased morbidity and comorbidities, such as the series of urological complications that impact quality of life [2]. Depending on the lesion level SCI causes detrusor-sphincter-dyssynergia with high leak point pressures that result in increased bladder thickness and fibrosis. Fibrosis may adversely affect smooth muscle function and the capacity of the bladder to empty properly, resulting in low capacity, high pressure, and noncompliance [3,4]. Bladder abnormalities are reportedly accompanied not only by changes in the bladder wall morphology, but also by drastic changes in the mechanical properties of the wall. Although extensive studies were conducted on the effects of SCI on bladder function, the alterations in mechanical properties of the bladder wall tissue and the underlying mechanisms are not yet well-understood [5]. Due to an increasing awareness of the above-described severe anatomical and functional LUT impairment after SCI and the lack of effective treatment options, interest in the research of LUT physiology and pathophysiology has increased. Much of the current knowledge is based on animal experimentation. Hence, species differences may be a problem when extrapolating animal findings to the human situation [6]. However, pigs have been long used as an animal model for the investigation of LUT function since many years and the anatomical, physiological, and pharmacological characteristics of their LUT is considered more similar to that of human than those of other animals, which have been studied extensively [7-10]. However, a detailed description of the changes of the urinary bladder of pigs after SCI is lacking to date. Therefore, the current study analysed the pathological changes of the urinary bladder in a minipig model of complete spinal cord transection. Hence, this study will give insights into the pathomechanisms Post-SCI in a translational animal model with value for subsequent minipig studies and possible future treatment strategies for patients.

\section{MATERIALS AND METHODS}

This study was carried out under the protocols approved by the
Ethics and Deontology Committee for Research on Animals, Victor Babes University of Medicine, Timisoara, Romania and were in compliance with the Association for Assessment of Laboratory Animal Care guidelines for animal use.

Seven healthy Göttingen Minipigs (females, 6 months of age, 18-30 kg; Ellegaard Minipigs, Dalmose, Denmark) were included in the study group. Bladder tissues of further 6 healthy minipigs (females, 4-8 months of age, $15-25 \mathrm{~kg}$ ) were used as healthy references to compare the structural outcomes. A Duragesic (Fentanyl transdermal system) patch $(100 \mu \mathrm{g} / \mathrm{kg}$ patch) was applied 24 hours prior to surgery. For antibiotic coverage, a single shot of Cefuroxime and Metronidazol was given prior to surgery. The minipigs were anesthetized by a cocktail containing Xylazine $(2 \mathrm{mg} / \mathrm{kg})$ and Ketamine $(15 \mathrm{mg} / \mathrm{kg})$ intravenously. Prior to intubation animals received Thiopentalum $(7 \mathrm{mg} / \mathrm{kg})$. General anesthesia was maintained by Sevoflurane $1.5 \%-2.5 \%$ delivered in a mixture of air/oxygen 1:1.

After induction of the general anaesthesia, the minipigs were placed in prone position and a Foley catheter (10 Charrière) was inserted for urine drainage during surgery. After skin incision, the skeletal muscle layers were transected to access the thoracic cord Th11 to Th13. A laminectomy was performed at the Th11-Th13 vertebral level. The spinal cord exposed at a length of $2 \mathrm{~cm}$. The epidural sack was carefully opened and epidural fatty tissue removed. The dura was carefully opened at a length of $1.5 \mathrm{~cm}$ and by means of bipolar electro-cauterization, spinal cord tissue was bluntly transected and removed to create a gap of $1 \mathrm{~cm}$ between the rostral and caudal endings of the spinal cord at Th12/Th13. Thereafter, the dura mater was sutured and fibrin glue was placed on the suture. The skeletal muscle and skin was closed in layers. Postsurgery, the minipigs were kept in single pens to avoid injury and stress. Twenty-four-hour surveillance and a tight timetable were followed to monitor the general health state and the lesion site. Antibiotic and analgesic coverage was continued for 5 days postoperative.

The follow-up time for all SCI minipigs was 4 months postSCI. During that time, a daily bladder diary was filled out for each SCI minipig. The bladder diary contained a daily urine strip test (Combur 10 test, Roche, Vienna, Austria) to count the specific weight, $\mathrm{pH}$, leukocytes, nitrite, protein, ketone bodies and blood cells (erythrocytes, haemoglobin). The permanent catheter was removed on day 5. Consecutively, the urinary bladder was drained every 4 hours by intermittent single catheterization for the duration of the follow-up time. Single catheterisation volumes as well as the total daily urine volume were 
noted in the bladder diary.

Motor function: Recovery of motor function was assessed using the 14-point scoring system (porcine neurological motor score) after Navarro et al. [11] Using this system (1) movement in all 3 joints in the lower extremities and tail, with no weight support, and (2) the degree of recovery of ambulatory function, with weight support could be assessed [11]. Testing of motor function was performed after 4 and 16 weeks of follow-up time in the open field and on a treadmill by 2 experienced investigators to define the ultimate porcine motor score scoring.

Sensory function: Sensory function was assessed by the presence of the withdrawal response to a mechanical stimulus. For this purpose, the minipigs were placed in a hammock. The toes of the front and hind limbs were progressively compressed with Halsted forceps. If sensation was present, a vigorous withdrawal response and/or vocalization was observed and the stimulus was immediately stopped. The response was scored as present or absent after 4 and 16 weeks follow-up time.

Muscle hypertonia: The presence of muscle hypertonia was defined as a spontaneous (stimulus-independent) increase in muscle tone. A positive response was assigned when the partially or fully paralyzed extremity was extended or flexed in the knee or hip joint, and returned to its original position.

Animals were sacrificed 4 months postoperative. The urinary bladder was removed and 2 samples of each urinary bladder wall were taken for microscopical analysis from the midbody of the bladder wall between bladder dome and trigone at a size of $2 \mathrm{~cm} \times 2 \mathrm{~cm}$. The 2 samples were taken from the most distant regions, fixed in ice-cold, phosphate-buffered $4 \%$ paraformaldehyde solution for 12 hours at $4^{\circ} \mathrm{C}$ and finally postprocessed for analyses.

For urinary bladder examination, the specimens were embedded in paraffin and serially sectioned at $10 \mu \mathrm{m}$. For analysis, the sections were histologically stained and examined (Axioplan fluorescence microscope, Zeiss, Oberkochen, Germany). For general urinary bladder wall analysis, a Masson-Goldner trichrome staining was utilized.

A number of 5 sections/specimen each $100 \mu \mathrm{m}$ from each other was chosen for analysis. The whole sections were photographically documented with slight overlap between single images at a magnification of $\times 20$ followed by qualitative and quantitative analyses. A stereological analysis that has been described elsewhere [12] was used to quantify the contents of urothelial, connective, smooth muscle, and vascular tissue. For this purpose, a digital 144-mesh grid, initially mesh size adjust- ed to the smallest structure, was used to quantify the tissues by means of ImageJ analysis software (National Institutes of Health, Bethesda, MD, USA). The stereological parameter employed for estimating the different tissue contents was volumetric density $(\mathrm{Vv})$. Mean values of $\mathrm{Vv}$ were calculated in relation to urothelial, connective, smooth muscle, and vascular tissue.

For elastic fibre examination a combined Elastica-Haematoxylin staining was used. A number of 5 sections/specimen in distance of $100 \mu \mathrm{m}$ to each other was chosen for analysis. For each of the 2 specimens per minipig's bladder, a series of 14 consecutive, nonoverlapping $\times 60$-magnified images of the suburothelial/detrusor region starting with the appearance of the first elastic fibres in the suburothelial area. Elastic fibres were digitally evaluated by rating the content elastic fibres/region (suburothelial or detrusor smooth muscle region) from 0-3 ( 0 , no elastic fibres; 1 , low content; 2 , moderate content; 3 , high content) by 2 persons. The ratings of the elastic fibres contents per region (suburothelial vs. detrusor smooth muscle) where then compared to each other and the means calculated. Additionally, the structural appearance of the elastic fibres was noted at high magnifications $(\times 60-\times 100)$.

To determine the amount of total collagen, naive sections were analyzed at $\times 20$ magnification with a polarisation filter using an ImageJ-based software. Total collagen was calculated by determining the area of collagen, detected by the polarisation filter. For the differentiation of types I and III collagen, a combined Haematoxylin and Herovici's staining protocol was applied and a spectrum analysis by means of Image J was conducted. For this purpose, the red stained type I collagen fibres were quantified and opposed to the quantified blue stained type III collagen fibres. All the above-described analyses were also conducted for the healthy reference urinary bladder tissues of the age-matched minipigs.

Comparisons between the 2 groups were made by nonparametric Mann-Whitney testing with 95\% confidence interval. A $\mathrm{P}$-value of $<0.05$ was considered significant and a $\mathrm{P}<0.001$ as highly significant.

\section{RESULTS}

\section{Routine Follow-up}

None of the SCI minipigs died during SCI surgery or during the 4-month follow-up period and no severe infections or injuries were observed. All SCI minipigs showed stable severe neurological deficits (grade 0) during follow-up. Complete paraple- 
gia was seen in all SCI animals according to the complete transection of the spinal cord at thoracic level Th12. All animals showed a baseline increase in muscle tone and spastic tail movements, starting 4 weeks after SCI. Sensory response was normal for the front limbs, while hind limbs response was absent during follow-up in all minipigs.

All minipigs tolerated single intermittent catheterization well. No urethral lesion, bleeding or procedural difficulties occurred. Urine strip tests showed no urinary tract infection as indicated by leucocyte/erythrocyte positive tests with or without the presence of nitrite. Events of intermittent isolated leucocyturia were noted, but were not confirmed by repeated tests. Bladder capacities and total daily urine excretion varied widely, but a trend towards smaller bladder volumes during the 4-month follow-up period (Fig. 1) and an increased number of incontinence periods was observed.

\section{Changes in the Bladder Wall Thickness and Its Composition After SCl}

After SCI the bladder wall thickness increased significantly $(\mathrm{P}<0.001)$ compared to healthy controls from average $0.42 \mathrm{~cm}$ to $0.83 \mathrm{~cm}$. Furthermore, the urinary bladder wall underwent drastic changes in its composition (Fig. 2). In comparison to healthy control urinary bladder tissue, a significant loss of smooth muscle tissue and correlated significant increase of connective tissue was seen. There were no distinct changes in the contents of urothelial tissue and blood vessels (Fig. 3).
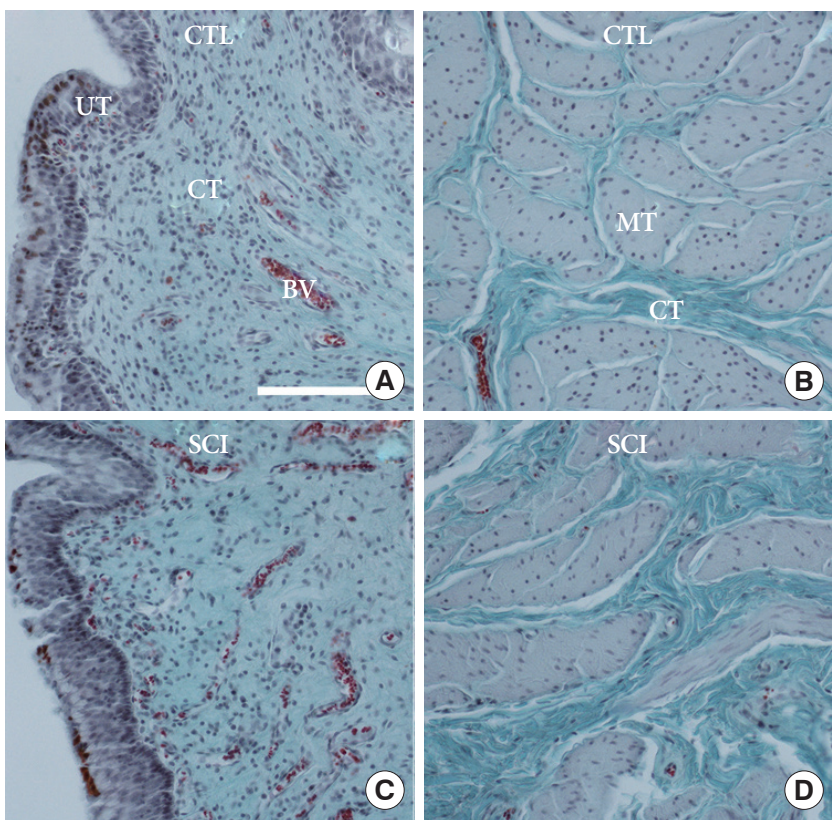

Fig. 2. (A) Comparison of the urinary bladder wall of a selected healthy reference minipigs (CTL) to a selected spinal cord injury (SCI) minipig. (A, C) Urothelial and suburothelial region of the urinary bladder wall. (B, D) Detrusor smooth muscle region of the urinary bladder wall. BV, blood vessel; CT, connective tissue; MT, smooth muscle tissue; UT, urothelial tissue. Masson-Goldner staining. The scale bars represent $1 \mathrm{~mm}$.
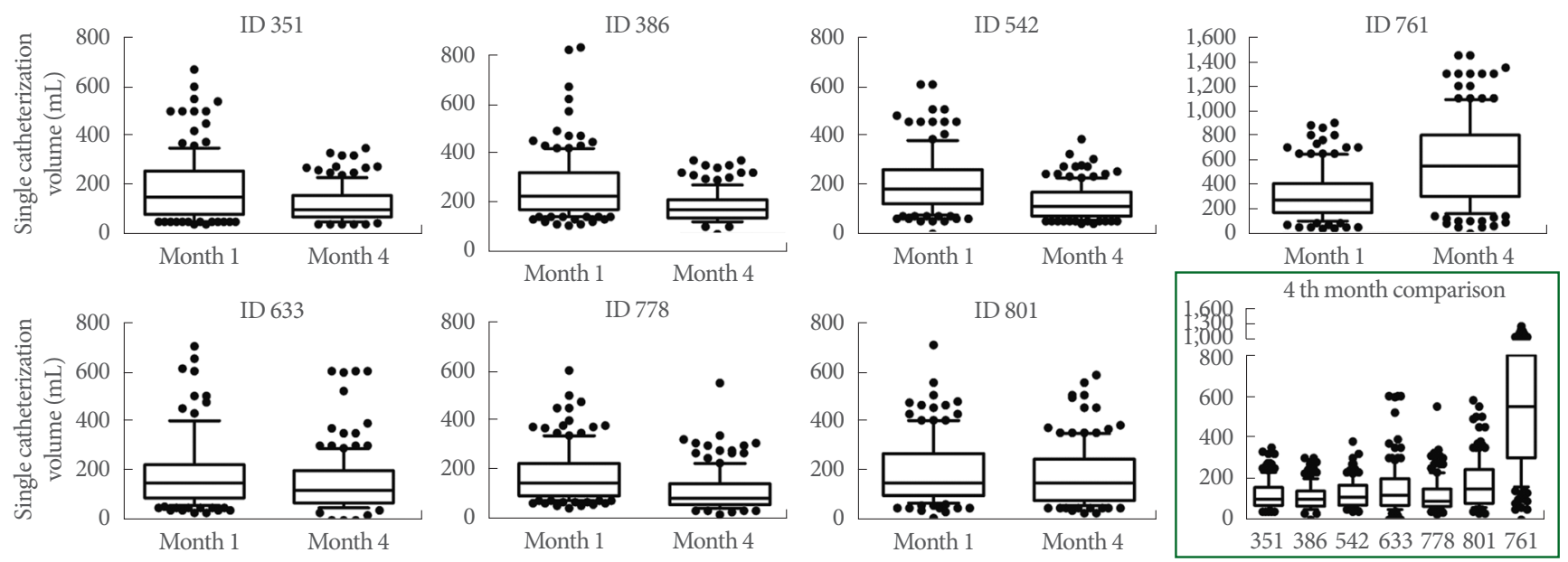

Fig. 1. Single catheterization volumes during months 1 and 4 post spinal cord injury (SCI) follow-up for the single seven SCI minipigs. Box plot displays the median (line) and the lower and upper quartiles. The whiskers show percentiles from $10 \%$ to $90 \%$. Outliers are seen by the single dots. The $\mathrm{x}$-axis shows the months 1 and 4 . The $\mathrm{y}$-axis depicts the single catheterisation volume (mL). The lower right image (confined by green lines) shows an assembly of the interanimal single catheterization volumes at month 4 . The $\mathrm{x}$-axis shows the single animal IDs. The y-axis displays the single catheterisation volume ( $\mathrm{mL})$. 


\section{Alterations of the Elastic Fibres After SCI}

Elastic fibres were reduced of up to $50 \%$ in SCI minipigs in comparison to healthy controls. The biggest loss of elastic fibres was observed in the smooth muscle tissue. Only 2 out of 7 minipigs showed light or moderate declines, whilst the others displayed higher grades of reduction.

Moreover, structural alterations amongst the elastic fibres were observed (Fig. 4). Elastic fibres in smooth muscle tissue lost their corkscrew-like shape and showed a reduction in di-

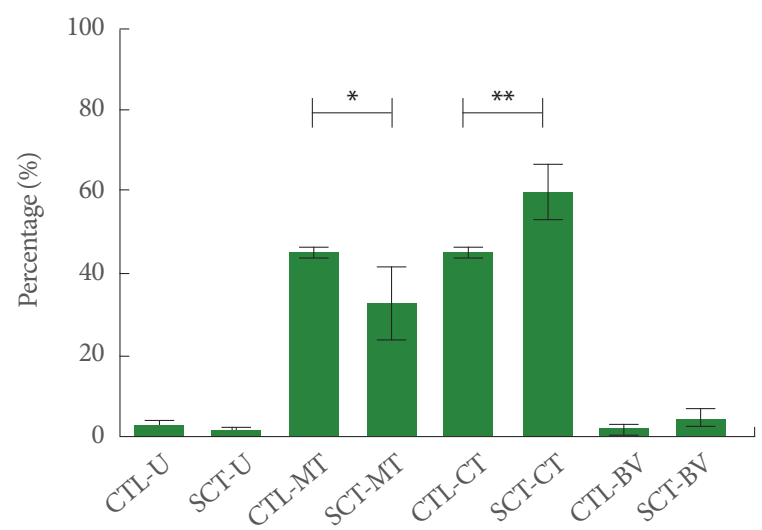

Fig. 3. Quantitative measurement of urinary bladder wall tissues of healthy reference minipigs (CTL) and spinal cord injury minipigs (SCT). The urothelium (U), smooth muscle (MT), connective (CT), and vascular tissue (VT) were analysed. MT showed a significant loss ( $\left.{ }^{*} \mathrm{P}=0.046\right)$, while CT showed a highly significant increase $\left({ }^{* *} \mathrm{P}=0.006\right)$. $\mathrm{X}$-axis: single tissues per group; $\mathrm{Y}$-axis: Percentages. Values are presented as mean \pm standard deviation. ameter and length. Elastic fibres lying in the extracellular matrix (ECM) compartment showed strong reductions in diameter and length. Often, only short fragments of elastic fibres were found.

\section{Total Collagen and Differentiation of Types I and III Collagen After SCI}

Collagen enclosure was mainly seen in the ECM of smooth muscle tissue (Fig. 5A) after SCI and total collagen ratio increased significantly in the ECM post-SCI (Fig. 5B). When differentiating between types I and III collagen, type I collagen enclosure was mainly seen in the smooth muscle tissue, while type III collagen increases were mainly seen in the suburothelial connective tissue (Fig. 6A). Post-SCI, a significant shift towards type I collagen was seen (Fig. 6B).

\section{DISCUSSION}

The aim of this study was to analyse structural changes of the urinary bladder after complete SCI during 4 months of followup in adult female Göttingen minipigs to establish a large animal model and describe the observations of relevant changes.

Routine management of our minipigs provided good health states, which is essential for a structural analysis. Based on an extensive study on the routine follow up of SCI minipigs by Zurita et al. [13], we established and further adapted a comprehensive health monitoring program which was proven to be effective to maintain good health states in our animals. Urinary tract infec-
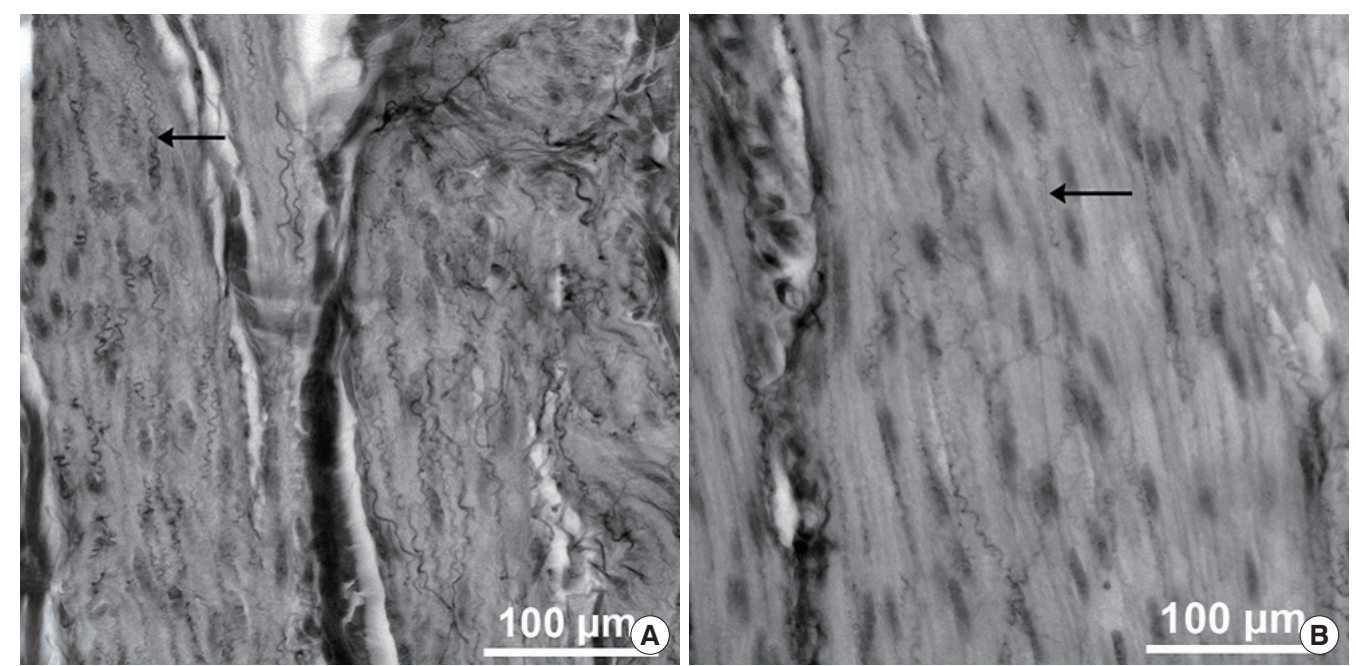

Fig. 4. Elastic fibres (arrows) in smooth muscle tissue of the urinary bladder of healthy reference minipigs (A) in comparison to spinal cord injury minipigs (B). Combined Elastica-Haematoxylin staining. The scale bars represent $100 \mu \mathrm{m}$. 

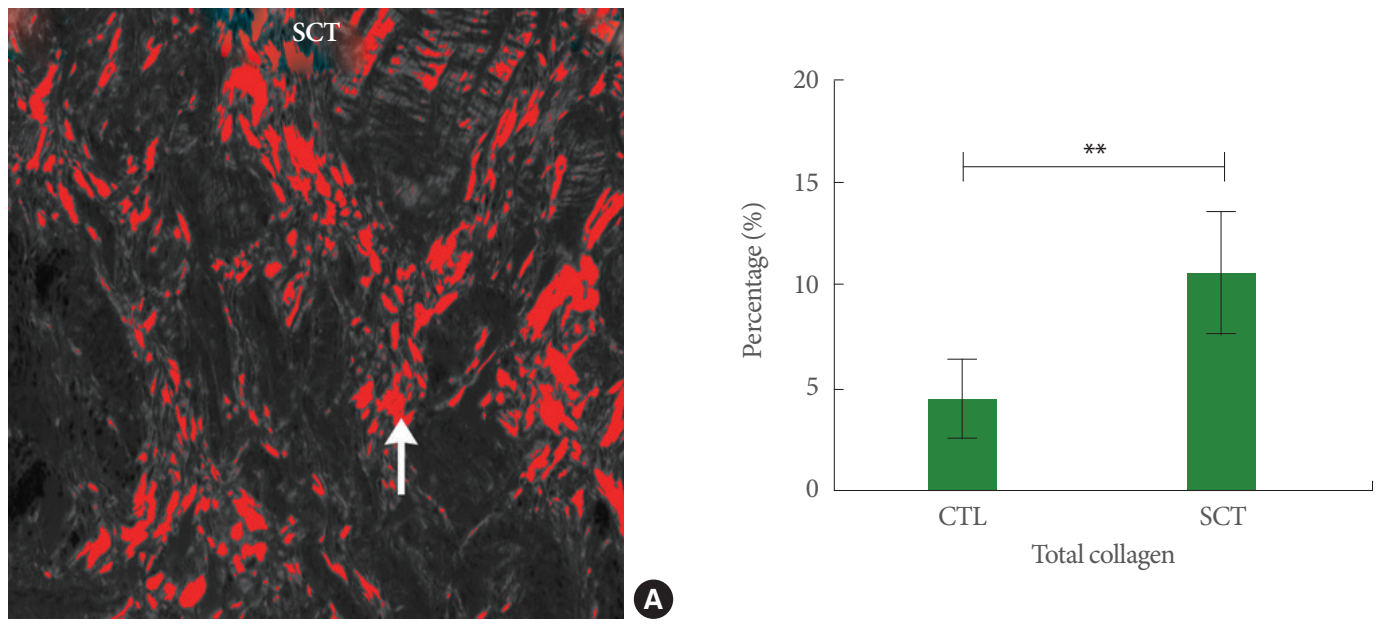

B

Fig .5. (A) Image showing the collagen amount in the extracellular matrix of the detrusor smooth muscle region of a spinal cord injury minipig (SCT). The area in red depicts the digital overlap of the detected collagen content (arrow) in a naive section. Magnification: $\times 14.4$. (B) Total collagen in healthy reference minipigs (CTL) and SCT. A highly significant difference was observed $\left({ }^{* *} \mathrm{P}<0.0001\right)$. Xaxis: groups; Y-axis: percentage of total collagen (\%). Values are presented as mean \pm standard deviation.
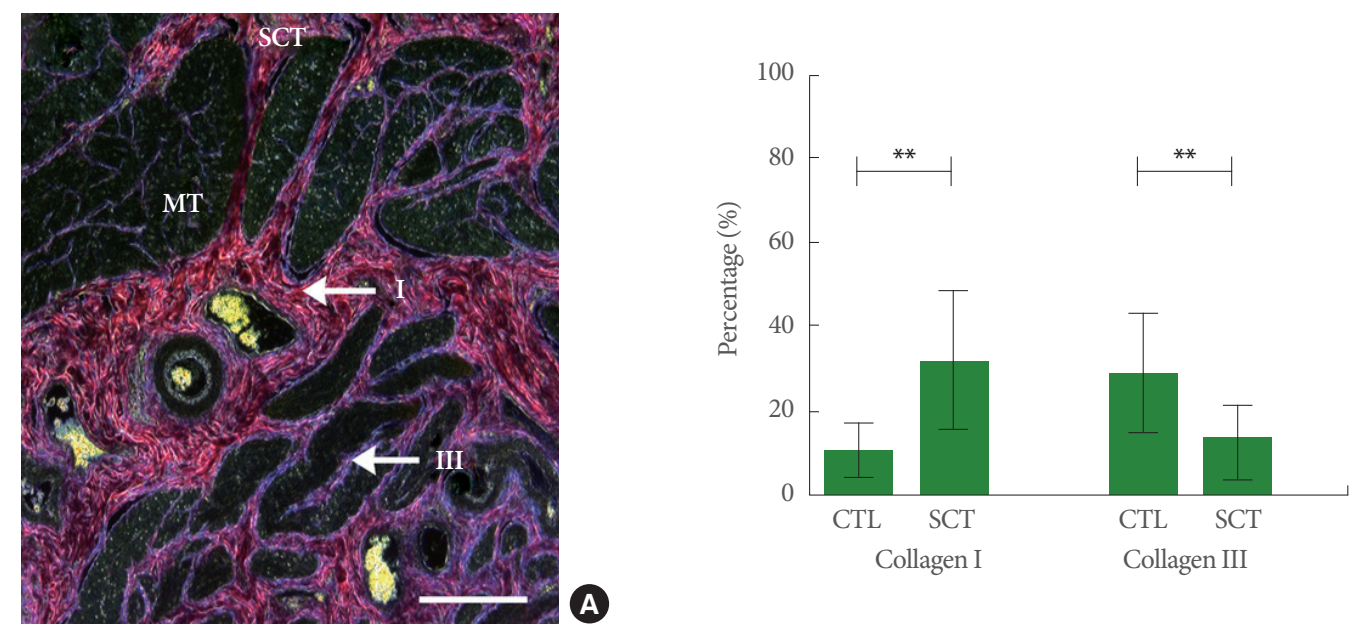

Fig. 6. (A) Detrusor smooth muscle region of a spinal cord injury minipig (SCT) with red stained type I collagen and blue stained type III collagen fibres in the extracellular matrix photographed with a special polarization filter. Combined Haematoxylin and Herovici's staining protocol. The scale bar represents $2.5 \mathrm{~mm}$. (B) Types I and III collagen percentage in healthy reference minipigs (CTL) and SCT. The difference between types I and III collagen between CTL and SCT are both highly significant $\left({ }^{* *} \mathrm{P}<0.0001\right)$. X-axis: groups; Y-axis: percentage of either collagen I or III (\%). Values are presented as mean \pm standard deviation. MT, smooth muscle tissue.

tions were not a relevant influencing factor, neither under clinical aspects, nor in the dipsticks. This is in contrast to the chronic SCI minipig study, in which a urinary tract infection rate of $20 \%$ was seen in their minipigs during follow-up after SCI [13]. The provided 24/7-hour care and the intermittent catheterisation (IC) seemed to be effective to prevent urinary tract infections. Thus, we consider IC as an integral element of the Post-SCI minipig care. Furthermore, the bladder diary outcomes with the trend towards smaller bladder volumes are important parameters for the later structural interpretation of data and underline the changes in bladder function over time post-SCI. Moreover, the amount of liquid intake and the extent of incontinence episodes are important parameters to consider. However, they can hardly be monitored properly during the follow-up time. This represents a limitation of our study as we could not integrate a permanent bladder monitoring system into our set up. 
The bladder wall consists mainly of collagen, elastin, and smooth muscles in addition to urothelium, nerves, and blood vessels [14]. Collagen is a source of tensile strength for the tissues, whereas elastin is essential for matrix resilience [15]. It has been suggested that the ECM has an effect on the passive properties of the bladder wall and that collagen and elastic fibres may also play an important role in the transmission of intercellular active force [16]. Therefore, a change in the ECM composition may also affect contractile properties of smooth muscles. In patients with chronic SCI, bladders will become hypertrophic and/or fibrotic while compliance of the bladder is significantly altered compared with normal bladder [3]. Structural changes were observed in all the above-described tissues.

The enlargement of the lamina propria, which forms a relatively thick layer of fibroelastic connective tissue allowing considerable distention and incorporating numerous blood vessels, was mainly due to an increase in collagen fibrils and an increase in ECM space. Elastic fibres were still present in the lamina propria, however, their shape was altered and the fibres were in clearer distance. Collagen type III remained the dominant collagen type in the lamina propria in both, SCI and healthy minipigs.

The muscular wall, the largest compartment within the urinary bladder wall, showed the most significant differences after SCI. In a healthy urinary bladder, the detrusor contains a large number of smooth muscle cells with ECM tissue for functional support. After SCI, the number of smooth muscle cells was significantly reduced. Further, the amount of elastic fibres was strongly decreased and vice versa the collagen content was significantly increased. With regard to the collagen types, there was a significant shift from type III to type I. All alterations, smooth muscle reduction, collagen increase/type shift and elastin loss, indicated a bladder wall fibrosis that may lead to a reduced functionality of the entire bladder. Types I and III collagen appeared to be the most abundant collagen types that significantly influence biological functions of the LUT. Type I collagen provides tensile strength of the tissue, while type III collagen is often associated with muscle fibres [17]. Any abnormality of the ratio of these 2 collagen types with a shift towards the dominance of type I collagen has a profound impact on mechanical properties of connective tissue of the bladder. Finally, it ends up in decreased contractility of the smooth muscles and, thus, a lack of bladder compliance [17-20]. Thus, this needs further confirmation by a functional study, as the whole picture can only be drawn by a combination of both, structural and functional analyses. Yet, the hereby described structural altera- tions give an insight on the obviously severe alterations of the urinary bladder after SCI which also results in changes in bladder function as it is known from patients with SCI.

In conclusion, this extensive structural analysis of the urinary bladder of minipigs after chronic SCI, highlighted that the urinary bladder wall undergoes fibrotic changes as seen by a strong increase in bladder wall thickness and a correlated increase of connective vs. decrease of smooth muscle tissue. Moreover, a trend towards reduced contractility and elasticity can be hypothesised based on the type I collagen increase and type III collagen/elastin decreased. These changes show in detail how SCI severely influences and alters the urinary bladder wall structure in minipigs and reflects the similarities between minipigs and humans. The study outcomes will help in future to better understand and interpret functional clinical aspects.

\section{ACKNOWLEDGEMENTS}

The authors wish to thank Professor Karl Dietrich Sievert (University Clinics of Urology and Andrology, Paracelsus Medical University) and Professor Sébastien Couillard-Després, (Department of Experimental Neuroregeneration, Spinal Cord Injury and Tissue Regeneration Center Salzburg, Paracelsus Medical University), for their valuable contributions. Furthermore, we thank the Spinal Cord Injury and Tissue Regeneration Center Salzburg Core Facilities Imaging and Histology for excellent technical support. We also thank Adrian Zeltner from Ellegaard Göttingen Minipigs for excellent support. We are grateful to the Pius Branzeu Center for Flap Surgery and Microsurgery, especially to Bogdan Hoinoiu, PhD. We thank Dan Negoescu, MD, $\mathrm{PhD}$, and Marcel Angelescu, MD, from the County Emergency Hospital "Pius Brinzeu" Timisoara, Politrauma Compartment and Neurosurgical Department - Casa Austria for their excellent advices during the Spinal Cord Surgeries. We would like to express our appreciation to the team of the Horia Cernescu Animal Facility of the Banat University of Agriculture and Veterinary Sciences, Timisoara, Romania, for their excellent care of the minipigs. Finally, we thank Patricia Toomey for editorial comments.

\section{REFERENCES}

1. de Groat WC. Mechanisms underlying the recovery of lower urinary tract function following spinal cord injury. Paraplegia 1995; 33:493-505. 
2. Cruz CD, Cruz F. Spinal cord injury and bladder dysfunction: new ideas about an old problem. ScientificWorldJournal 2011;11:21434.

3. Chen G, Liao L, Dong Q, Ju Y. The inhibitory effects of pudendal nerve stimulation on bladder overactivity in spinal cord injury dogs: is early stimulation necessary? Neuromodulation 2012;15: 232-7.

4. Lee HJ, An J, Doo SW, Kim JH, Choi SS, Lee SR, et al. Improvement in spinal cord injury-induced bladder fibrosis using mesenchymal stem cell transplantation into the bladder wall. Cell Transplant 2015;24:1253-63.

5. Toosi KK, Nagatomi J, Chancellor MB, Sacks MS. The effects of long-term spinal cord injury on mechanical properties of the rat urinary bladder. Ann Biomed Eng 2008;36:1470-80.

6. Andersson KE, Arner A. Urinary bladder contraction and relaxation: physiology and pathophysiology. Physiol Rev 2004;84:93586.

7. Jensen KN, Deding D, Sørensen JC, Bjarkam CR. Long-term implantation of deep brain stimulation electrodes in the pontine micturition centre of the Göttingen minipig. Acta Neurochir (Wien) 2009;151:785-94.

8. Mills IW, Drake MJ, Greenland JE, Noble JG, Brading AF. The contribution of cholinergic detrusor excitation in a pig model of bladder hypocompliance. BJU Int 2000;86:538-43.

9. Dalmose AL, Bjarkam CR, Djurhuus JC. Stereotactic electrical stimulation of the pontine micturition centre in the pig. BJU Int 2005;95:886-9.

10. Bossowska A, Crayton R, Radziszewski P, Kmiec Z, Majewski M. Distribution and neurochemical characterization of sensory dorsal root ganglia neurons supplying porcine urinary bladder. J Physiol Pharmacol 2009;60 Suppl 4:77-81.
11. Navarro R, Juhas S, Keshavarzi S, Juhasova J, Motlik J, Johe K, et al. Chronic spinal compression model in minipigs: a systematic behavioral, qualitative, and quantitative neuropathological study. J Neurotrauma 2012;29:499-513.

12. Weibel ER, Kistler GS, Scherle WF. Practical stereological methods for morphometric cytology. J Cell Biol 1966;30:23-38.

13. Zurita M, Aguayo C, Bonilla C, Otero L, Rico M, Rodríguez A, et al. The pig model of chronic paraplegia: a challenge for experimental studies in spinal cord injury. Prog Neurobiol 2012;97:288-303.

14. Madersbacher S, Pycha A, Klingler CH, Mian C, Djavan B, Stulnig $\mathrm{T}$, et al. Interrelationships of bladder compliance with age, detrusor instability, and obstruction in elderly men with lower urinary tract symptoms. Neurourol Urodyn 1999;18:3-15.

15. Montes GS. Structural biology of the fibres of the collagenous and elastic systems. Cell Biol Int 1996;20:15-27.

16. Susset JG, Regnier CH. Viscoelastic properties of bladder strips: standardization of a technique. Invest Urol 1981;18:445-50.

17. Kim JC, Yoon JY, Seo SI, Hwang TK, Park YH. Effects of partial bladder outlet obstruction and its relief on types I and III collagen and detrusor contractility in the rat. Neurourol Urodyn 2000;19:2942.

18. Lepor H, Sunaryadi I, Hartanto V, Shapiro E. Quantitative morphometry of the adult human bladder. J Urol 1992;148(2 Pt 1):4147.

19. Ewalt DH, Howard PS, Blyth B, Snyder HM 3rd, Duckett JW, Levin $\mathrm{RM}$, et al. Is lamina propria matrix responsible for normal bladder compliance? J Urol 1992;148(2 Pt 2):544-9.

20. Deveaud CM, Macarak EJ, Kucich U, Ewalt DH, Abrams WR, Howard PS. Molecular analysis of collagens in bladder fibrosis. J Urol 1998;160:1518-27. 